\title{
Kapillares Saugen durch eine Grenzfläche
}

\section{P. Prim}

Ehemals Laboratoire des matériaux de construction

EPF, Lausanne, Schweiz

\section{Zusammenfassung}

Zunächst wurde an Ziegelstein, grauem Sandstein, zementgebundenem Normenmörtel, Zement-Kalk-Mörtel und an Gips die kapillare Saugfähigkeit bestimmt. Aus diesen Komponenenten wurden zusammengesetzte Systeme gefertigt. Die Ergebnisse an den Systemen Ziegelstein/Normenmörtel und Sandstein/Gips werden beispielhaft beschrieben und diskutiert. Es zeigt sich, dass die Grenzfläche eine leicht bremsende Wirkung auf die kapillare Saugfähigkeit des nachgeschalteten Werkstoffes ausübt. Dies ist bei der Vorhersage der Saugfähigkeit von Mauerwerk basierend auf der gemessenen Saugfähigkeit der Komponenten zu berücksichtigen. Stichwörter: Kapillare Wasseraufnahme, poröse Werkstoffe, Grenzfläche

\section{Capillary suction through an interface}

\begin{abstract}
Capillary suction of burnt clay brick, sandstone, cement based standard mortar, cement-lime-mortar, and gypsum plaster has been determined experimentally. Composite systems have been made of these individually investigated components. Results of the systems burnt clay brick/standard mortar and sandstone/gypsum plaster are presented and discussed. It has been observed that the interface between two porous materials slightly reduces capillary suction of the second component. This effect has to be taken into consideration if capillary suction of masonry is to be predicted on the basis of measured values of the components.
\end{abstract}

Key words: Capillary suction, porous materials, interface 


\section{Einleitung}

Alle porösen Werkstoffe des Bauwesens nehmen Wasser durch kapillares Saugen auf, wenn ihre Oberfläche in Kontakt mit flüssigem Wasser kommt. Das kann durch Schlagregen, durch aufstehendes Wasser oder durch die Feuchtigkeit im Boden verursacht werden. Das eindringende Wasser ist häufig das Transportmittel für unterschiedliche Schadstoffe. Ausserdem wird durch hohe Feuchtigkeit die thermische Leitfähigkeit der Werkstoffe erhöht. Bei aufsteigender Feuchtigkeit können Bauteile durch den Kristallisationsdruck, der aus der aufkonzentrierten Lösung ausfallenden Salze nachhaltig beschädigt werden (siehe z.B. [1]).

Es besteht also ein elementares Interesse, das Saugverhalten von porösen Werkstoffen und von Bauteilen, die aus unterschiedlichen porösen Werkstoffen zusammengesetzt sind, zu kennen und, wenn immer nötig, zu begrenzen. Die kapillare Saugfähigkeit einzelner Komponenten lässt sich vergleichsweise rasch durch einen standardisierten Versuch bestimmen. Wie verhalten sich einzelne Komponenten aber im Mauerwerk und welchen Einfluss haben die Grenzflächen auf die Saugfähigkeit eines Verbundbauteiles?

In diesem Beitrag soll zunächst die kapillare Saugfähigkeit einzelner Komponenten wie Ziegelstein, Sandstein und unterschiedliche Mörtel beschrieben werden. Danach werden Ergebnisse, die an kleinen Verbundkörpern, bestehend aus zwei unterschiedlichen Werkstoffen, gewonnen wurden, dargestellt.

\section{Die untersuchten Proben}

Für eine erste Versuchsserie wurden Prismen mit den folgenden Abmessungen hergestellt: $40 \times 40 \times 160 \mathrm{~mm}$. Die Stirnflächen mit $40 \times 40 \mathrm{~mm}$ wurden zur Bestimmung der kapillaren Saugfähigkeit $1 \mathrm{~mm}$ tief in Wasser getaucht. Insgesamt wurden fünf unterschiedliche Werkstoffe in die Untersuchungen einbezogen:

1. Gebrannter Ziegel; (ZI)

2. Grauer Sandstein (Villarlod bleue); (SS)

3. Zementgebundener Normenmörtel; (NM)

4. Zement-Kalk-Mörtel (verlängerter Mörtel); (ZK)

5. Gips; (GI)

Die Zusammensetzung des Zementmörtels, des Zement-Kalk-Mörtels und des Gipsputzes ist in Tabelle I aufgeführt.

Aus gebranntem Ton (Ziegelstein) und grauem Sandstein einerseits und den drei unterschiedlichen Mörteln andererseits wurden insgesamt sechs zusammengesetzte Proben hergestellt. Die unterschiedlichen und untersuchten Kombinationsmöglichkeiten sind in Tabelle II gezeigt. Die Steinproben hatten jeweils die Abmessungen $40 \times 40 \times 40 \mathrm{~mm}$ und auf diese wurde eine $20 \mathrm{~mm}$ dicke Mörtelschicht aufgetragen. 
Alle Ergebnisse werden in einem internen Bericht zusammengestellt [2].

Tabelle I: Zusammensetzung der unterschiedlichen verwendeten Mörtel Table I Composition of the different mortars used in this investigation

\begin{tabular}{|l|c|c|c|c|c|c|}
\hline & $\begin{array}{l}\text { Normen- } \\
\text { sand } \\
0-5 \mathrm{~mm}\end{array}$ & $\begin{array}{l}\text { Portland- } \\
\text { zement }\end{array}$ & $\begin{array}{l}\text { Gelösch- } \\
\text { ter Kalk }\end{array}$ & Gips & Wasser & $\begin{array}{l}\text { Wasser/ } \\
\text { Bindmittel }\end{array}$ \\
\hline $\begin{array}{l}\text { Zementge- } \\
\text { bundener } \\
\begin{array}{l}\text { Normen- } \\
\text { mörtel }\end{array}\end{array}$ & $1350 \mathrm{~g}$ & $450 \mathrm{~g}$ & - & - & $198 \mathrm{~g}$ & 0.44 \\
\hline $\begin{array}{l}\text { Zement-Kalk- } \\
\text { Mörtel }\end{array}$ & $1 ' 350 \mathrm{~g}$ & $100 \mathrm{~g}$ & $250 \mathrm{~g}$ & - & $300 \mathrm{~g}$ & 0.85 \\
\hline Gips & - & - & - & $750 \mathrm{~g}$ & $300 \mathrm{~g}$ & 0.40 \\
\hline
\end{tabular}

Tabelle II: Kombinationsmöglichkeiten aus Stein und Mörtel für zusammengesetzte Proben

Table II: $\quad$ Possible combinations of stone and mortar for composite specimens

\begin{tabular}{|l|l|}
\hline \multicolumn{1}{|c|}{ Stein } & \multicolumn{1}{c|}{ Mörtel } \\
\hline \multirow{2}{*}{$\begin{array}{l}\text { Gebrannter Ton, } \\
\text { Ziegelstein }\end{array}$} & Zementgebundener Normenmörtel \\
\cline { 2 - 2 } & Zement-Kalk-Mörtel \\
\cline { 2 - 2 } $\begin{array}{l}\text { Grauer Sandstein } \\
\text { (Villarlod bleue) }\end{array}$ & Gips \\
\cline { 2 - 2 } & Zementgebundener Normenmörtel \\
\cline { 2 - 2 } & Zement-Kalk-Mörtel \\
\hline
\end{tabular}

\section{Ergebnisse}

3.1 Messungen an den Komponenten

Durch viele Versuche wurde nachgewiesen, dass sich der zeitliche Verlauf der kapillaren Wasseraufnahme $W(t)$ näherungsweise durch folgende einfache Gleichung beschreiben lässt:

$$
W(t)=A \sqrt{t}
$$

Darin steht A für den Koeffizienten der kapillaren Wasseraufnahme.

Die Wasseraufnahme als Funktion der Saugdauer ist in Abb. 1 graphisch dargestellt [2]. Aus der Steigung der Geraden lässt sich der Koeffizient der kapillaren Wasseraufnahme bestimmen. Die so erhaltenen Werte sind in Tabelle III zusammengestellt. Der zementgebundene Mörtel besitzt erwartungsgemäss nur eine geringe Saugfähigkeit. 


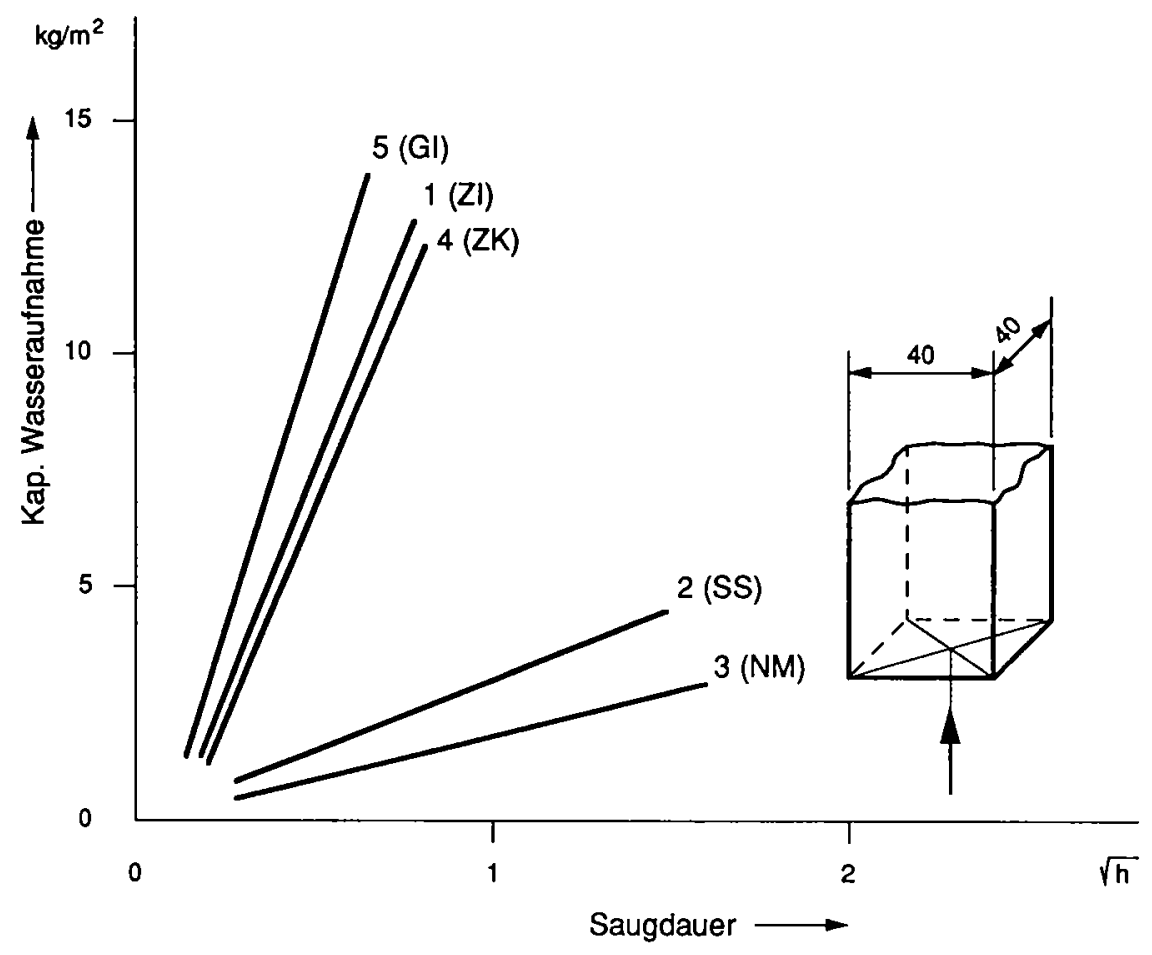

Abb. 1: Kapillare Wasseraufnahme der untersuchten Komponenten: 1. Gebrannter Ziegel (ZI), 2. Grauer Sandstein (SS), 3. Zementgebundener Normenmörtel (NM), 4. Zement-Kalk-Mörtel (ZK), 5. Gips (GI)

Fig. 1: Capillary suction of the samples investigated: 1. burnt clay (ZI), 2. Grey sandstone (SS), 3. Standard cement mortar (NM), 4. cement-lime-mortar (ZK), 5. Gypsum plaster (GI)

Tabelle III: Koeffizient A der kapillaren Wasseraufnahme der einzelnen Komponenten

Table III: Coefficient of capillary suction A of the different components

\begin{tabular}{|l|c|}
\hline \multicolumn{1}{|c|}{ Werkstoff } & $\mathrm{A} \frac{\mathrm{kg}}{\mathrm{m}^{2} \mathrm{~h}^{1 / 2}}$ \\
& 15.9 \\
\hline Gebrannter Ton & 2.9 \\
\hline Grauer Sandstein & 1.8 \\
\hline Normenmörtel & 14.7 \\
\hline Zement-Kalk-Mörtel & 20.3 \\
\hline Gips & \\
\hline
\end{tabular}




\subsection{Messungen an zusammengesetzten Proben}

An den zusammengesetzten Proben (siehe Tabelle II) wurde jeweils die kapillare Wasseraufnahme in zwei unterschiedlichen Richtungen A und B gemessen. Einmal wurde der Stein mit dem Wasser in Kontakt gebracht (Richtung A). Das Wasser wandert also über den Stein in den Mörtel. Zusätzlich wurde an identischen Proben die Wasseraufnahme in umgekehrter Richtung, also vom Mörtel in den Stein, gemessen.

In Abb. 2 sind die Ergebnisse der zusammengesetzten Proben aus Ziegelstein und Normenmörtel aufgetragen. Zum Vergleich sind die an den einzelnen Komponenten gemessenen und in Abb. 1 gezeigten Verläufe noch einmal gestrichelt eingezeichnet.

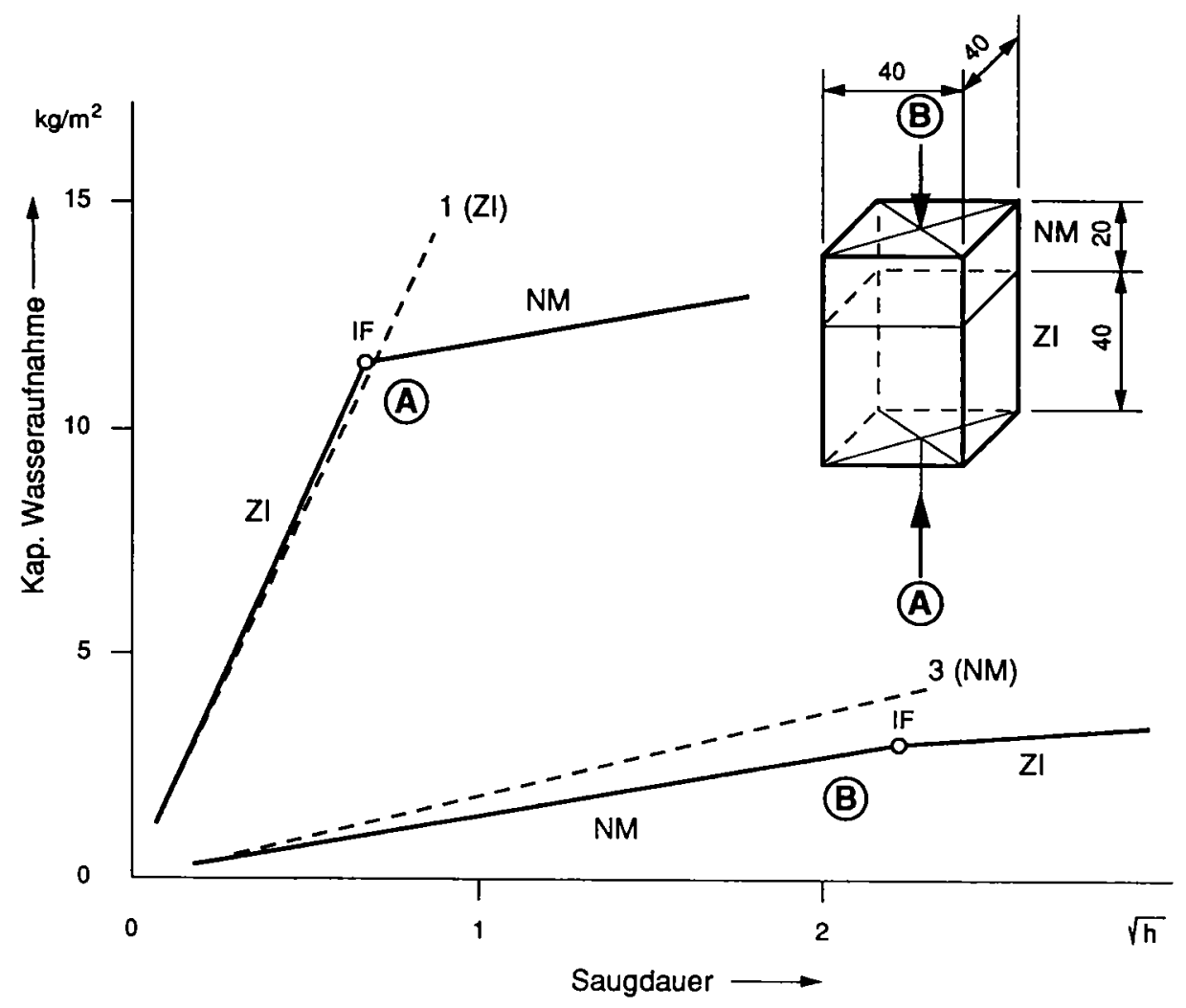

Abb. 2: Kapillare Wasseraufnahme des zusammengesetzten Systems Ziegelstein/ Normenmörtel: Richtung $A$ = über den Ziegel in den Normenmörtel und Richtung $\mathrm{B}=$ über den Normenmörtel in den Ziegel.

Fig. 2: Capillary suction of the composite system burnt clay/standard mortar, Direction $A=$ via burnt clay into the standard mortar and direction $B=$ via the standard mortar into the burnt clay. 
Im Rahmen der Messgenauigkeit stimmt die Wasseraufnahme des Ziegelsteins in der zusammengesetzten Probe mit dem Ergebnis der entsprechenden Messung an den Komponenten überein, wenn das Wasser über den Ziegelstein aufgenommen wird (Richtung A in Abb. 2). An der mit IF (interface) bezeichneten Stelle macht der Verlauf einen Knick. Zu diesem Zeitpunkt war die aufsteigende Wasserfront gerade an der Grenzfläche zwischen dem Ziegelstein und dem Zementmörtel angekommen. Die Saugfähigkeit des Mörtels ist anschliessend durch einen Koeffizienten $\mathrm{A}=1.2 \mathrm{~kg} / \mathrm{m}^{2} \mathrm{~h} 1 / 2$ charakterisiert und liegt damit geringfügig unter dem an dieser Komponente getrennt bestimmten Wert (vergleiche mit Tabelle III).

Im Rahmen der Messgenauigkeit wurde an dem zusammengesetzten System Ziegel/Kalk-Zement-Mörtel ein analoges Verhalten beobachtet. Beim System Ziegel/Gips dagegen wirkt die Grenzfläche in beiden Richtungen stärker als Bremse für die kapillare Saugfähigkeit der angrenzenden Komponente. Die beiden kapillaren Systeme sind im letztgenannten Fall sehr unterschiedlich; sie sind also nicht kompatibel.

Auch das Verhalten der zusammengesetzten Systeme Sandstein/Zementmörtel und Sandstein/Kalk-Zement-Mörtel ist dem oben beschriebenen Verhalten vergleichbar. In Abb. 3 sind noch die Ergebnisse, die am System Sandstein/Gips bestimmt wurden, graphisch dargestellt. Man sieht, dass der stark saugende Gips, wenn er dem Sandstein nachgeschaltet ist, gerade die angebotene Wassermenge weiterleitet (Richtung A). In umgekehrter Richtung stellt man zunächst wieder fest, dass der Gips vermutlich durch Wasserentzug durch den porösen Untergrund im Verbund mit dem Sandstein etwas weniger saugfähig ist als an den einzelnen Komponenten gemessen (siehe Abb. 1 und Tabelle III). Der nachgeschaltete Sandstein (Richtung B) saugt im System mit etwas reduzierter Saugkraft.

\section{Folgerungen}

Es wurde gezeigt, dass die Grenzfläche zwischen zwei porösen Werkstoffen eine geringe Bremswirkung auf die kapillare Saugfähigkeit ausübt. Dies kann vermutlich damit erklärt werden, dass eine aufgebrachte Putz- bzw. Mörtelschicht teilweise in die oberflächennahen Poren des Untergrundes eindringt. Dadurch wird der Übergang des Wassers von einem porösen System ins andere behindert.

Wenn in Saugrichtung eine stark saugende Komponente auf eine wenig saugende folgt, so kann die stark saugende fast alles angebotene Wasser weitertransportieren. Folgt in Saugrichtung dagegen eine weniger saugende Komponente auf eine stark saugende, so übernimmt die schwach saugende nur noch einen Teil des angebotenen Wassers. 


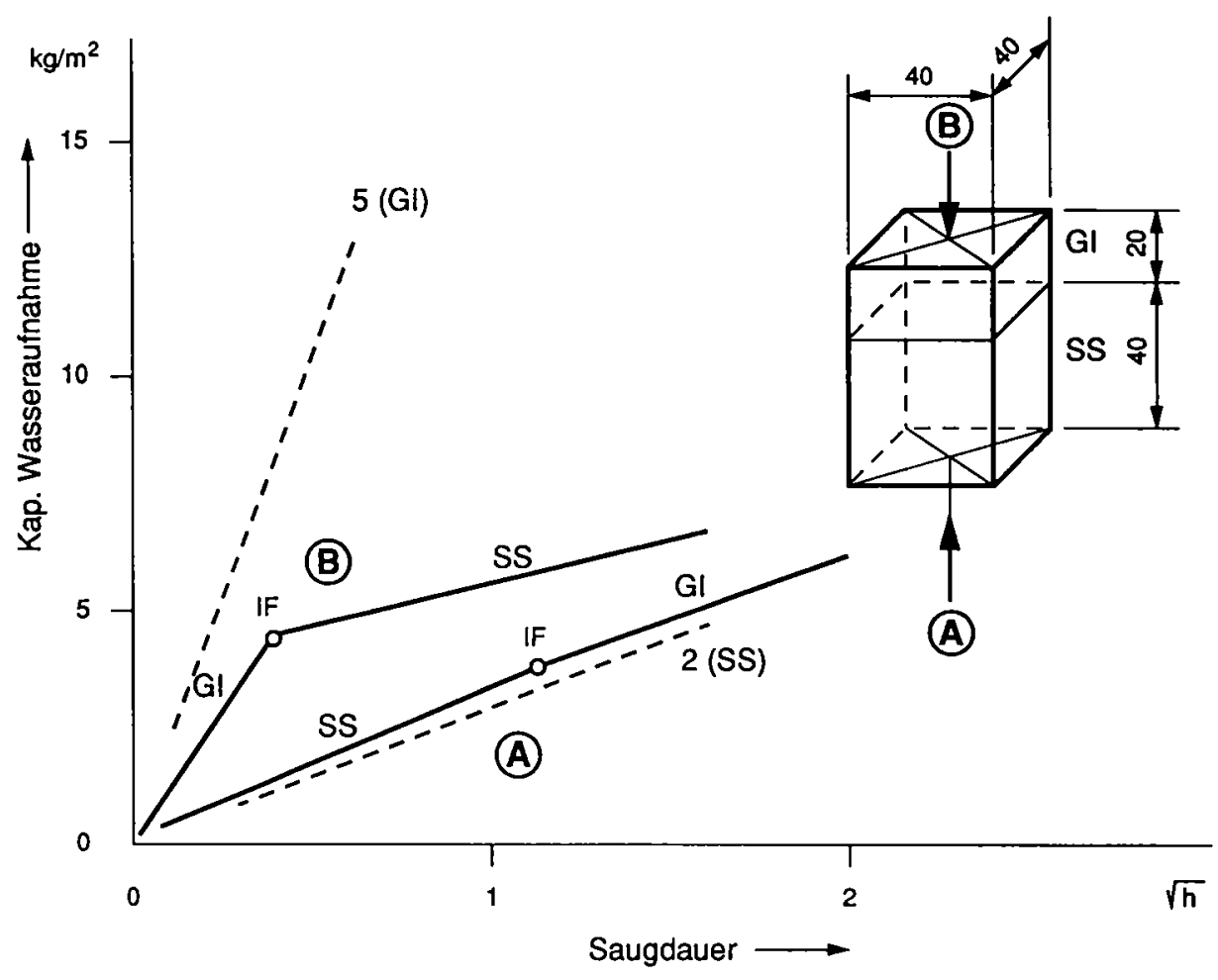

Abb. 3: Kapillare Wasseraufnahme des zusammengesetzten Systems Grauer Sandstein/Gipsputz: Richtung A = über den Sandstein in den Gipsputz und Richtung B = über den Gipsputz in den Sandstein.

Fig. 3: Capillary suction of the composite system sandstone/gypsum plaster: direction $a=$ via the sandstone into the gypsum plaster and dirction $B=$ via the gypsum plaster into the sandstone.

\section{Literatur}

1. G. Mayer und F.H. Wittmann, Wasser- und Salztransport bei aufsteigender Feuchtigkeit, Veröffentlichung angenommen in Int. Z. Bauinstandsetzen (1996).

2. P. Prim, Capillarité des matériaux de construction - influence d'une interface, LMC, EPFL, Lausanne, interner unveröffentlichter Bericht (1988) 
